\title{
Multifocus confocal Raman microspectroscopy for fast multimode vibrational imaging of living cells
}

\author{
Masanari Okuno ${ }^{1}$ and Hiro-o Hamaguchi ${ }^{1,2, *}$ \\ ${ }^{1}$ Department of Chemistry, School of Science, University of Tokyo, 7-3-1 Hongo, Bunkyo-ku, Tokyo, Japan, 113-0033 \\ ${ }^{2}$ Institute of Molecular Science and Department of Applied Chemistry, National Chiao Tung University, \\ 1001 Ta Hsueh Road, Hsinchu 300, Taiwan \\ *Corresponding author: hhama@chem.s.u-tokyo.ac.jp
}

Received August 26, 2010; revised October 12, 2010; accepted October 20, 2010;

posted October 21, 2010 (Doc. ID 134062); published December 2, 2010

\begin{abstract}
We have developed a multifocus confocal Raman microspectroscopic system for the fast multimode vibrational imaging of living cells. It consists of an inverted microscope equipped with a microlens array, a pinhole array, a fiber bundle, and a multichannel Raman spectrometer. Forty-eight Raman spectra from 48 foci under the microscope are simultaneously obtained by using multifocus excitation and image-compression techniques. The multifocus confocal configuration suppresses the background generated from the cover glass and the cell culturing medium so that high-contrast images are obtainable with a short accumulation time. The system enables us to obtain multimode (10 different vibrational modes) vibrational images of living cells in tens of seconds with only $1 \mathrm{~mW}$ laser power at one focal point. This image acquisition time is more than 10 times faster than that in conventional singlefocus Raman microspectroscopy. () 2010 Optical Society of America

OCIS codes: $110.0180,120.6200,290.5860,300.6450$.
\end{abstract}

Raman microspectroscopy is now well established as a unique and powerful method for investigating living cells in vivo [1]. The only drawback of this method is its slow speed owing to low cross sections of Raman scattering. It often takes a few to tens of minutes to obtain a Raman image of a living cell. If we want to increase the speed, we need to increase the excitation laser power. However, higher laser power causes photo and/or thermal damage of the cell. Empirically, laser power higher than several milliwatts causes obvious cell damage. This constraint can be circumvented by exciting the sample with multiple laser foci, whose intensities are below the damage threshold. In this Letter, we report the development of a multifocus confocal Raman microspectroscopic system that achieves fast vibrational imaging of living cells in tens of seconds. The multifocus excitation technique has already been used in other microscopies: single [2]/two-photon [3] fluorescence microscopy, coherent anti-Stokes Raman scattering (CARS) microscopy [4], and second-harmonic generation microscopy [5]. $\overline{\mathrm{By}}$ combining multifocus excitation and the image compression technique [6,7], we have realized multifocus confocal Raman microspectroscopy, which simultaneously provides the spatial and spectral information on different chemical compounds in a living cell. Raman spectral information enables us to make multimode vibrational images due to different Raman bands as well as to discriminate Raman signals from the background arising from the cover glass and the cell culturing medium.

Figure 1 shows the diagram of our multifocus confocal Raman microspectroscopic system. The expanded beam of an $\mathrm{Nd}: \mathrm{YVO}_{4}$ laser (Coherent, Verdi; $532 \mathrm{~nm}$ ) illuminates a microlens array (MLA) of $147 \mu$ m diameter and $4.9 \mathrm{~mm}$ focal length on a fused-silica disk. The beamlets are collimated by lenses and are reflected by an edge filter that reflects the $532 \mathrm{~nm}$ excitation light but transmits Raman scattered light with longer wavelengths. The beamlets then pass a pinhole array (Ø $100 \mu \mathrm{m}, 200 \mu \mathrm{m}$ pitch, $8 \times 8$ rectangular pattern) and intermediate optics and are introduced into an inverted microscope (Nikon, modified ECLIPSE Ti-U). The role of the intermediate optics is to ensure that the array of foci is imaged onto the focal plane of the lens and that each beamlet is parallel at the objective entrance pupil. The objective lens (Nikon $\times 100$ NA 1.3 oil immersion) produces a pattern of $8 \times 8$ independent foci at the sample. Raman signals from the foci at the sample are collimated by the same objective lens. The Raman signals go back and pass through the pinhole array. The role of the pinhole array is to improve the axial resolution and to enhance the ratio between the Raman signal and background by the confocal effect [8]. This multiconfocal configuration is indispensable for obtaining high-contrast images of living cells in which weak Raman signals are easily overwhelmed by the background arising from the cover glass and cell culturing medium. The other important role of the pinhole array is to eliminate the unwanted laser light that goes through the MLA without being collimated. The Raman signals pass through filters to eliminate Rayleigh scattering and are collimated into the one end of a fiber bundle, which guides the signals into a spectrograph. The fiber bundle consists of 64 fibers, whose core, clad, and jacket are $\varnothing 100 \mu \mathrm{m}, \varnothing 120 \mu \mathrm{m}$, and $\varnothing 140 \mu \mathrm{m}$, respectively. The 64 fiber bundle is arranged in a $8 \times 8$ rectangular

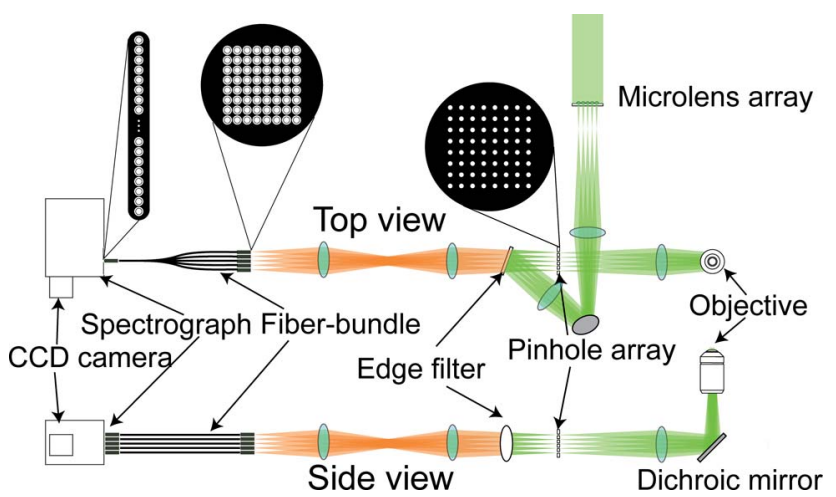

Fig. 1. (Color online) Schematic (top and side views) of the multifocus confocal Raman microspectroscopic system. 


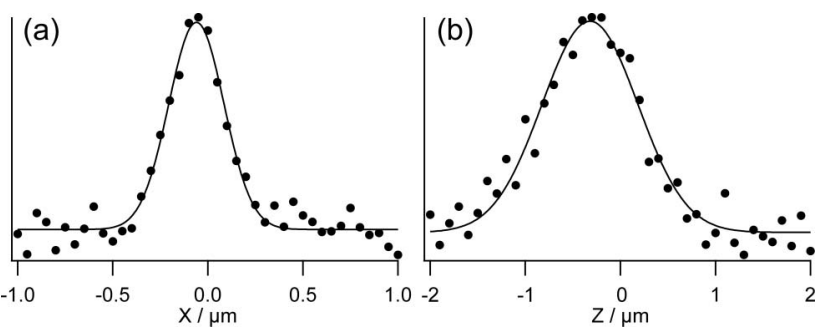

Fig. 2. (a) Lateral and (b) depth profiles of a single polystyrene bead with $356 \mathrm{~nm}$ diameter.

pattern at the collection end and a $1 \times 64$ linear stack at the detection end, which is connected to the entrance slit of the spectrograph directly. Using this fiber bundle, a three-dimensional [two-dimensional (2-D) spatial and spectral] data cube is converted into a 2-D data array, which can be detected by a 2-D CCD camera. Finally, the Raman signals are dispersed in the spectrograph (HORIBA Jobin Yvon, iHR320) and detected by a CCD camera (Princeton Instruments, ProEM512). In the present setup, 48 spectra from 48 points $(X$ : eight points $Y$ : six points) are detectable with one CCD exposure.

At the sample position, two adjacent foci are $2.0 \mu \mathrm{m}$ apart in the lateral direction with $\mathrm{a} \times 100$ objective. We fill this gap of $2.0 \mu \mathrm{m}$ by $4 \times 4$ point-by-point sample scanning by a piezo stage (Mad City Labs, Nano-LPQ). The resultant Raman image consists of $32 \times 24$ points covering the $16 \mu \mathrm{m} \times 12 \mu \mathrm{m}$ area. The spectral resolution, $\sim 10 \mathrm{~cm}^{-1}$ in the fingerprint region, is determined by the dispersion of the spectrograph and the core size of the fiber bundle. The spectral coverage in one exposure is $\sim 600 \mathrm{~cm}^{-1}$.

We measured a single $356 \pm 20 \mathrm{~nm} \emptyset$ polystyrene bead on a glass-bottom dish in order to evaluate the spatial resolution of the system. The intensity profiles of the Raman band at $1603 \mathrm{~cm}^{-1}(\mathrm{C}=\mathrm{C}$ stretch) are shown in Figs. 2(a) (lateral) and 2(b) (axial). The FWHMs are $340 \mathrm{~nm}$ and $1.2 \mu \mathrm{m}$, respectively. These values are very similar to those obtained in conventional single-focus confocal Raman microspectroscopy. This fact indicates that each focus is collimated by the objective lens close to the diffraction limit and that the pinhole array acts as a spatial filter eliminating the out-of-focus signal by the confocal effect.

Next, budding yeast cells (a zygote of Saccharomyces cerevisiae and Saccharomyces bayanus) were measured. Figure 3 shows a CCD image with $10 \mathrm{~s}$ exposure time. The image consists of the 48 Raman spectra from the 48 foci at the sample. The correspondence between the spectrum number on the CCD image and the sample position under the microscope is shown in Fig. 3(b). To compensate the variation of the laser intensity and the signal collection efficiency among the 48 foci, the image is corrected by the fluorescence of a Rhodamine 6G ethanol solution. Figures 3(b) and 3(c) compare the Raman spectra extracted from two different horizontal bands in Fig. 3(a). These two spectral profiles are totally different from each other. It is confirmed that Raman signals from two different points of the sample are separately detected simultaneously by the CCD camera.

The Raman images of budding yeast cells are shown in Fig. 4. In this experiment, budding yeast cells were immobilized on a concanavalin-A-coated glass-bottom dish.
The total laser power at the sample is $\sim 70 \mathrm{~mW}$, so the laser power at each focus is approximately $1 \mathrm{~mW}$. It is obvious that a $\sim 70 \mathrm{~mW}$ single focus totally destructs living cells. The distribution of the total laser power into multiple foci significantly reduces the photodamage. The exposure time for one series of 48 spectra is $1 \mathrm{~s}$ and overall measurement time is $20 \mathrm{~s}$. $1 \mathrm{~s}$ exposure time for 48 spectra is equivalent to less than $25 \mathrm{~ms}$ for one spectrum. It is not possible to obtain a high signal-to-noise-ratio Raman spectrum in such a short exposure time by conventional Raman microspectroscopy. Ten multimode Raman images due to 10 different Raman bands are simultaneously obtained, including those at 1446 [Fig. 4(a)], 1583 [Fig. 4(b)], 1602 [Fig. 4(c)], and $1655 \mathrm{~cm}^{-1}$ [Fig. 4(d)]. The Raman bands at 1446, 1583, and $1655 \mathrm{~cm}^{-1}$ are assigned to the $\mathrm{CH}$ bend, the porphyrin in-plane $\mathrm{C}=\mathrm{C}$ stretch mode of the porphyrin skeleton of cytochrome c [9], and the superposition of the cis $-\mathrm{C}=\mathrm{C}$ stretch of unsaturated lipid chains and the amide I mode of proteins, respectively. The $1602 \mathrm{~cm}^{-1}$ Raman band, called the "Raman spectroscopic signature of life" by us, sharply reflects the metabolic activity of a living cell $[10,11]$. Figure 4(c) shows a highly localized distribution of the $1602 \mathrm{~cm}^{-1}$ band, which overlaps partially with the images of the 1446 and $1655 \mathrm{~cm}^{-1}$ Raman bands. On the other hand, Fig. 4(b) shows a totally different distribution of cytochrome $\mathrm{c}$ from the other three bands. The similarity and variation among the four images originate from the different distributions of different chemical compounds giving rise to different Raman bands. It should be emphasized that two yeast cells are
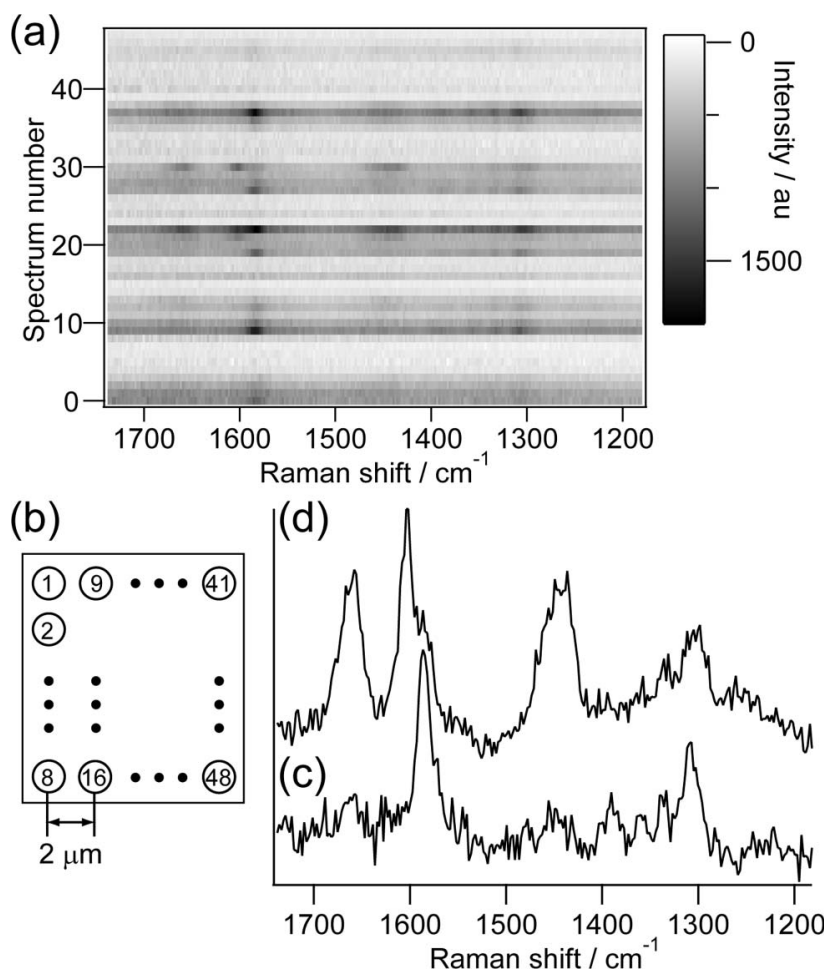

Fig. 3. (a) CCD image consisting of 48 Raman spectra of budding yeast cells. (b) Correspondence between the spectrum number in (a) and the position of the focus under the microscope. Extracted Raman spectra at (c) 27 and (d) 30 in (a). Spectra are intensity-corrected by the fluorescence spectra of a Rhodamine $6 \mathrm{G}$ ethanol solution. 


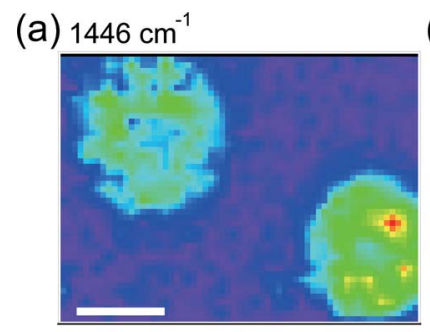

(b) $1583 \mathrm{~cm}^{-1}$

(c) $1602 \mathrm{~cm}^{-1}$

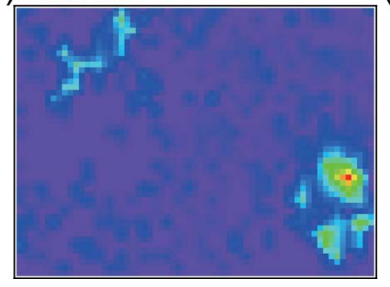

(d) $1655 \mathrm{~cm}^{-1}$

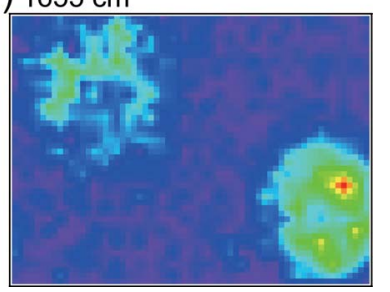

Fig. 4. (Color online) Raman images of budding yeast cells of the Raman bands at (a) 1446, (b) 1583, (c) 1602, and (d) $1655 \mathrm{~cm}^{-1}$. The total acquisition time is approximately $20 \mathrm{~s}$. The scale bar in the image is $4 \mu \mathrm{m}$.

simultaneously Raman imaged. It takes several tens of minutes to make a Raman image of such a wide area $(16 \mu \mathrm{m} \times 12 \mu \mathrm{m})$ by conventional confocal Raman microspectroscopy.

It is interesting to compare multifocus confocal Raman microspectroscopy with multiplex CARS microspectroscopy. The previous multiplex CARS studies show that a multiplex CARS spectrum of polymer sample is obtained within $340 \mu$ s to $100 \mathrm{~ms}$ [12-14]. A spontaneous Raman spectrum of polystyrene can be obtained in several to $10 \mathrm{~ms}$, including the CCD readout time in the present system. These results suggest that the Raman imaging speed of our system is comparable to that of multiplex CARS microspectroscopy [15].

In conclusion, we have constructed a multifocus confocal Raman microspectroscopic system. Multimode Raman images originating from different vibrational modes of different chemical compounds can be obtained with a high efficiency owing to the simultaneous acquisition capability of both the spectral and spatial information. It is more than 10 times faster than the conventional confocal Raman microspectroscopy. Multifocus confocal Raman microspectroscopy is a promising tool for studying living cells in vivo and in real time.

This work is supported by the System Development Program for Advanced Measurement and Analysis (Program-S) of the Japan Science and Technology Agency (JST). We are grateful to Dr. F. Okura and Ms. H. Yomo (Suntory Co., Ltd.) for providing us with the yeast sample.

\section{References}

1. G. J. Puppels, F. F. M. Demul, C. Otto, J. Greve, M. Robertnicoud, D. J. Arndtjovin, and T. M. Jovin, Nature 347, 301 (1990)

2. T. Tanaami, S. Otsuki, N. Tomosada, Y. Kosugi, M. Shimizu, and H. Ishida, Appl. Opt. 41, 4704 (2002).

3. J. Bewersdorf, R. Pick, and S. W. Hell, Opt. Lett. 23, 655 (1998).

4. T. Minamikawa, M. Hashimoto, K. Fujita, S. Kawata, and T. Araki, Opt. Express 17, 9526 (2009).

5. M. Kobayashi, K. Fujita, T. Kaneko, T. Takamatsu, O. Nakamura, and S. Kawata, Opt. Lett. 27, 1324 (2002).

6. A. D. Gift, J. Y. Ma, K. S. Haber, B. L. McClain, and D. Ben-Amotz, J. Raman Spectrosc. 30, 757 (1999).

7. J. Y. Ma and D. Ben-Amotz, Appl. Spectrosc. 51, 1845 (1997).

8. G. J. Puppels, W. Colier, J. H. F. Olminkhof, C. Otto, F. F. M. Demul, and J. Greve, J. Raman Spectrosc. 22, 217 (1991).

9. S. Z. Hu, I. K. Morris, J. P. Singh, K. M. Smith, and T. G. Spiro, J. Am. Chem. Soc. 115, 12446 (1993).

10. Y. S. Huang, T. Karashima, M. Yamamoto, T. Ogura, and H. Hamaguchi, J. Raman Spectrosc. 35, 525 (2004).

11. Y.S. Huang, T. Karashima, M. Yamamoto, and H. Hamaguchi, Biochemistry 44, 10009 (2005).

12. T. W. Kee and M. T. Cicerone, Opt. Lett. 29, 2701 (2004).

13. M. Cui, B. R. Bachler, and J. P. Ogilvie, Opt. Lett. 34, 773 (2009).

14. J. Sung, B. C. Chen, and S. H. Lim, "Fast three-dimensional chemical imaging by interferometric multiplex coherent anti-Stokes Raman scattering microscopy," J. Raman Spectrosc. (to be published).

15. M. Okuno, H. Kano, and H. Hamaguchi are preparing a manuscript to be called "Raman and CARS: which wins in living cell spectroscopy and imaging?" 